\title{
Rapamycin downregulates thymidylate synthase and potentiates the activity of pemetrexed in non-small cell lung cancer
}

\author{
Shigeru Kawabata ${ }^{1, *}$, Chun-Te Chiang 2,* , Junji Tsurutani ${ }^{2}$, Hideaki Shiga 2,3, Matthew \\ L. Arwood ${ }^{1}$, Takefumi Komiya², Joell J. Gills', Regan M. Memmott ${ }^{1}$, and Phillip A. \\ Dennis ${ }^{1}$ \\ ${ }^{1}$ Department of Oncology, Johns Hopkins Bayview Medical Center, Baltimore, MD, USA \\ ${ }^{2}$ Medical Oncology Branch, Center for Cancer Research, National Cancer Institute, Bethesda, MD, USA \\ ${ }^{3}$ Current address: Department of Otorhinolaryngology, Kanazawa Medical University, Ishikawa, Japan \\ * These authors contributed equally to this work \\ Correspondence to: Phillip A. Dennis, email: pdennis@jhmi.edu \\ Keywords: Rapamycin, Pemetrexed, Drug synergy, mTOR, Thymidylate Synthase, Lung Cancer \\ Received: January 7, $2014 \quad$ Accepted: February 15, $2014 \quad$ Published: February 16, 2014
}

This is an open-access article distributed under the terms of the Creative Commons Attribution License, which permits unrestricted use, distribution, and reproduction in any medium, provided the original author and source are credited.

\section{ABSTRACT:}

Non-small cell lung cancer (NSCLC) accounts for $80-85 \%$ of lung cancer cases, and almost half of newly diagnosed patients have metastatic disease. Pemetrexed is a widely used drug for NSCLC and inhibits several folate-dependent enzymes including thymidylate synthase (TS). Increased expression of TS confers resistance to pemetrexed in vitro and predicts poor response to pemetrexed. Rapamycin is an mTOR inhibitor and suppresses cap-dependent synthesis of specific mRNA species. Here, we show that the combination of rapamycin and pemetrexed synergistically inhibits proliferation of NSCLC cells. Although pemetrexed as a single agent induced TS, pretreatment with rapamycin suppressed pemetrexed-induced TS expression. In vivo, the combination of rapamycin and pemetrexed inhibited growth of NSCLC xenografts, which correlated with decreased mTOR activity and suppression of pemetrexed-induced TS expression. The ability of rapamycin to enhance the efficacy of pemetrexed and prevent TS expression has implications for the design of Phase I and/or Phase II NSCLC clinical trials with mTOR inhibitors in combination with pemetrexed.

\section{INTRODUCTION}

Approximately 1.7 million new cancer cases and 0.6 million cancer deaths are projected to occur in the United States in 2013, and lung cancer is responsible for $26 \%$ and $28 \%$ of all female and male cancer deaths, respectively [1]. Non-small cell lung cancer (NSCLC) accounts for $80-85 \%$ of lung cancers, and $54 \%$ of patients with newly diagnosed NSCLC have advanced disease (i.e., distant stage or metastasis) [2]. Platinum-based chemotherapy regimens given as first-line treatment to advanced NSCLC patients with a good performance status have plateaued in overall response rate $(25 \%-35 \%)$, median survival (8-10 mo.), one-year survival $(30 \%-40 \%)$, and two-year survival (10\%-15\%) [3]. The best chemotherapy regimen for patients with advanced NSCLC remains to be determined.

Pemetrexed is a folate antimetabolite that is chemically similar to folic acid and inhibits three enzymes that contribute to purine and pyrimidine synthesis; thymidylate synthase (TS), hidydrofolate reductase (DHFR), and glycinamide ribonucleotide formyltransferase (GARFT) [4]. Pemetrexed was approved by the US Food and Drug Administration (FDA) as a firstline therapy for advanced non-squamous NSCLC patients when combined with cisplatin and as a maintenance therapy for patients with advanced non-squamous NSCLC who do not experience disease progression after platinum-based chemotherapy $[5,6]$. Pemetrexed as a single-agent is a standard treatment for recurrent NSCLC patients who have previously received platinum-based chemotherapy, but the overall response rate is less than $10 \%$ and resistance to pemetrexed eventually develops [4]. The modest responses in the front line, second line, and maintenance settings provide rationale to develop new 
approaches to enhance the efficacy of pemetrexed.

Several mechanisms of resistance to antifolates have been described that include impaired influx, alteration of intracellular metabolism, increased target enzymes, an expanded folate pool, and increased efflux [7]. Increased expression of TS confers resistance to pemetrexed in lung cancer cells in vitro $[8,9]$ and is a predictive factor for poor response to pemetrexed in patients [10], indicating that inhibition of TS expression may be beneficial for overcoming resistance to pemetrexed.

The mammalian target of rapamycin (mTOR) is a serine/threonine protein kinase that belongs to the phosphoinositide 3-kinase (PI3K)-related kinase family, and controls protein synthesis, energy metabolism, cell proliferation, and survival [11]. Recently, it has been reported that pemetrexed can activate AMP-activated protein kinase (AMPK) through inhibition of a folatedependent enzyme called aminoimidazolecarboxamide ribonucleotide formyltransferase (AICART), which indirectly inhibits mTOR and decreases phosphorylation of downstream substrates[12, 13], suggesting that mTOR inhibition can be a consequence of anti-folate activities.

Previously we reported that rapamycin, an mTOR inhibitor, prevented the development of tobacco carcinogen-induced lung tumors in a mouse model, suggesting that the mTOR pathway is implicated in lung tumorigenesis [14]. Because rapamycin has broad anti-proliferative activity across a series of NSCLC cell lines, we hypothesized that combining rapamycin with pemetrexed might enhance mTOR inhibition, suppress TS expression, and synergistically decrease proliferation of NSCLC cells. Our data confirm that rapamycin enhances the efficacy of pemetrexed and suppresses pemetrexedinduced TS expression in vitro and in vivo.

\section{RESULTS}

To assess effects on cellular proliferation, rapamycin and pemetrexed were tested at concentrations that are clinically achievable in a series of four NSCLC cell lines that vary in status of molecular targets such as EGFR and
Kras. The inhibitory concentration 50 (IC50) values of each drug when used in combination were less than when used as single treatments, indicating synergistic effects at the effective dose 50 (ED50) (Table 1). Computersimulated Fa-CI curves showed synergism (CIs $<1$ ) in the four cell lines evaluated (Figure 1 and Supplementary Table 1). These data suggest that rapamycin enhances the anti-proliferative effects of pemetrexed.

Because rapamycin is a prototypic mTOR inhibitor and pemetrexed has been reported to decrease mTOR activity in a small number of cell lines, mTOR inhibition was assessed in a series of NSCLC cell lines. Phosphorylation of the ribosomal protein S6 and 4E-BP1 was measured as a surrogate for mTOR activity. S6 is phosphorylated by S6K, which is a direct substrate of mTOR. 4E-binding protein 1 (4E-BP1) is regulated by mTOR and inhibits $5^{\prime}$-cap-dependent mRNA translation by binding and inactivating eukaryotic translation initiation factor 4E (eIF4E) that is involved in the mRNAribosome binding step of eukaryotic protein synthesis. Phosphorylated 4E-BP1 by mTOR is dissociated from eIF4E, resulting in increasing protein synthesis [11]. As a single agent, pemetrexed decreased phosphorylation of S6 and 4E-BP1 in H460, H157 and H1155 cells, but to a lesser extent than rapamycin as a single agent. Combining rapamycin with pemetrexed decreased phosphorylation of 4E-BP1 and increased hypophosphorylated forms of 4E$\mathrm{BP} 1$, but the effects of the combination on phosphorylation of S6 were less apparent because rapamycin was highly effective at inhibiting S6 phosphorylation as a single agent (Figure 2). These results suggested that the combination of rapamycin and pemetrexed enhances the inhibition of the mTOR pathway, but also raised the possibility that other molecular targets might be responsible for the combined anti-proliferative effects.

An increased basal level of TS expression is recognized as a mechanism of resistance to pemetrexed $[8,9]$. Pemetrexed has been reported to inhibit TS activity in NSCLC patients [15], but high concentrations of pemetrexed can induce TS expression in A549 cells [16]. To clarify the effects of pemetrexed on TS expression,

Table 1: IC50 values of pemetrexed and rapamycin in either single or the combination treatment.

\begin{tabular}{|c|c|c|c|c|c|c|}
\hline \multirow[b]{2}{*}{ Cell line } & \multirow[b]{2}{*}{ ExposureTime } & \multicolumn{2}{|c|}{ Single treatment } & \multicolumn{3}{|c|}{ Combination treatment } \\
\hline & & Pemetrexed & Rapamycin & Pemetrexed & Rapamycin & CI at ED50 \\
\hline & (hr) & $(\mathbf{n M})$ & $(\mathrm{nM})$ & $(\mathrm{nM})$ & $(\mathrm{nM})$ & \\
\hline $\mathrm{H} 460$ & 72 & 308.96 & 41.27 & 19.28 & 0.96 & 0.09 \\
\hline $\mathrm{H} 157$ & 96 & 77.16 & 2.51 & 58.36 & 0.23 & 0.85 \\
\hline H1975 & 96 & 581.56 & 4.03 & 52.23 & 1.04 & 0.35 \\
\hline H1155 & 72 & 73.03 & 1.43 & 42.06 & 0.42 & 0.87 \\
\hline
\end{tabular}

NSCLC cells were treated with $0.1 \%$ DMSO as a control or varying concentrations of pemetrexed, rapamycin, or the combination for cell proliferation assay and assessment of CI values as described in Materials and Methods. Inhibitory concentration 50 (IC50) is the concentration of an inhibitor where the response is reduced by half, whereas effective dose 50 (ED50) is the concentration of a drug that gives half-maximal response. Combination Index (CI) values $<1$ indicate synergism. See also Supplementary Table 1 . 
two concentrations of pemetrexed were used in four NSCLC cell lines (Figure 3A). Our results show that pemetrexed potently induced TS expression, because 100 $\mathrm{nM}$ increased TS expression in all four cell lines. To assess the effects of combining rapamycin and pemetrexed, simultaneous administration for $24 \mathrm{hr}$ and sequential administration (rapamycin for $24 \mathrm{hr}$ followed by the addition of pemetrexed for $24 \mathrm{hr}$ ) were compared (Figure 3B). As a single agent given for $24 \mathrm{hr}$, pemetrexed induced TS expression and modestly decreased phosphorylation of S6K in the three cell lines tested. Rapamycin as a single agent markedly decreased S6K phosphorylation in all cell lines tested, and modestly decreased the basal level
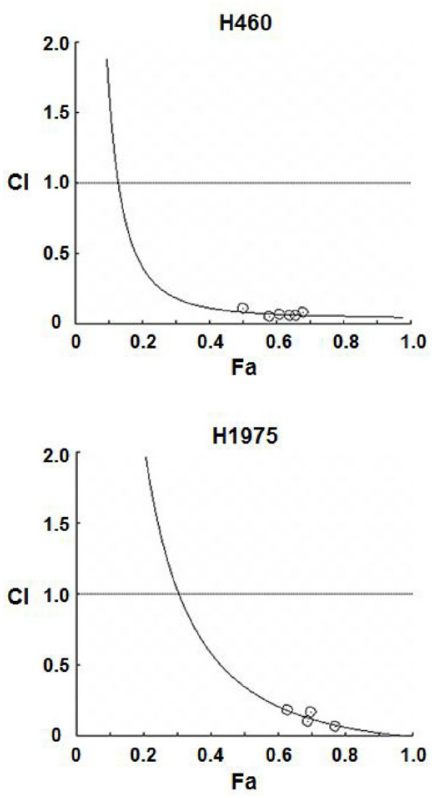

of TS in H157 cells. When rapamycin and pemetrexed were administered simultaneously, rapamycin only modestly decreased the expression of TS that was induced by pemetrexed, suggesting that pretreatment with rapamycin might prevent pemetrexed-induced TS expression to a greater extent. Indeed, pretreatment of cells with rapamycin substantially improved its ability to inhibit pemetrexed-induced increases in TS expression. Collectively, these findings suggest that rapamycin might increase the efficacy of pemetrexed by preventing pemetrexed-induced TS expression.

To determine if the combination of rapamycin and pemetrexed has enhanced anti-tumor effects, athymic
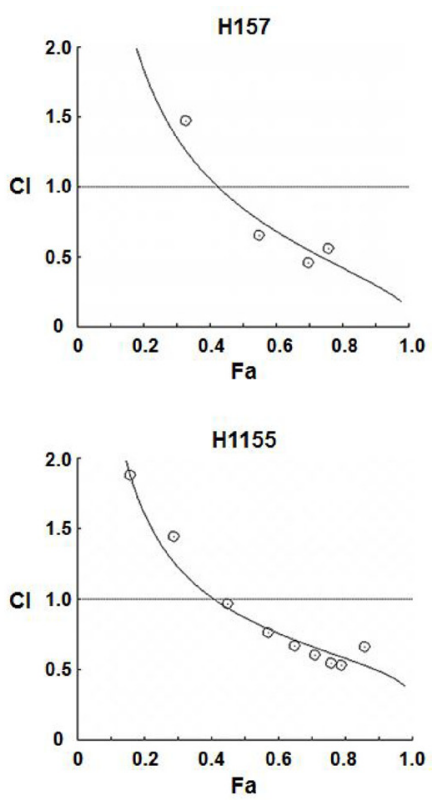

Figure 1: Pemetrexed and rapamycin synergistically inhibit the proliferation of NSCLC cells in vitro. NSCLC cells were treated with $0.1 \%$ DMSO or varying concentrations of pemetrexed, rapamycin, or the combination for cell proliferation assays and assessment of CI values as described in Materials and Methods. Computer-simulated Fa-CI curves show synergism $(\mathrm{CI}<1)$, additive effect $(\mathrm{CI}=1)$, or antagonism $(\mathrm{CI}>1)$ for the indicated levels of growth inhibition $(\mathrm{Fa})$ induced by the drug combination (see also Supplementary Table 1A). Circles (O) indicate the Fa-CI data points based on experimental values (see also Supplementary Table 1B).

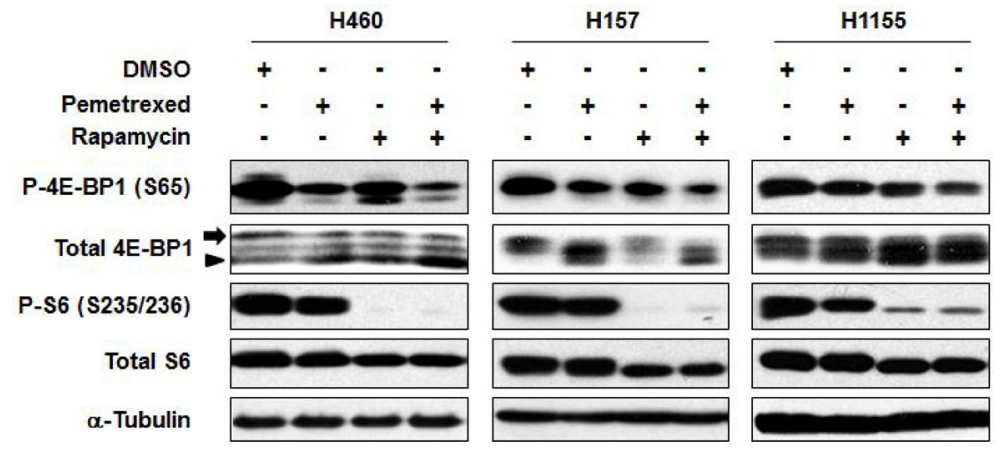

Figure 2: The combination of rapamycin and pemetrexed inhibits the mTOR pathway to a greater extent than either drug alone in a panel of NSCLC cell lines. IC50 concentrations were used for these studies. Specifically, H460 cells were treated with either $309 \mathrm{nM}$ pemetrexed, $41 \mathrm{nM}$ rapamycin, or the combination for $72 \mathrm{hr}$; H157 cells were treated with either $77 \mathrm{nM}$ pemetrexed, $2.5 \mathrm{nM}$ rapamycin, or the combination for $96 \mathrm{hr}$; and $\mathrm{H} 1155$ cells were treated with either $73 \mathrm{nM}$ pemetrexed, $1.4 \mathrm{nM}$ rapamycin, or the combination for $72 \mathrm{hr}$ (see also Table 1). As a control, the indicated cell lines were treated with $0.1 \%$ DMSO. Immunoblotting analysis was performed for components of the mTOR pathway. The arrow and arrowhead indicate hyper- and hypophosphorylated forms of 4E-BP1, respectively. 
$\mathrm{NCr}-\mathrm{nu} / \mathrm{nu}$ mice bearing established H460 (Figure 4BD) or H157 (Figure 4E-G) tumor xenografts were treated with either vehicle, $2.5 \mathrm{mg} / \mathrm{kg}$ rapamycin, $100 \mathrm{mg} /$ $\mathrm{kg}$ pemetrexed, or the combination (Figure 4A). The treatment schedule was designed to allow for a three day window of rapamycin pretreatment prior to simultaneous treatment. The combination was well tolerated and decreased $\mathrm{H} 460$ tumor growth by nearly $70 \%$, which was greater than inhibition due to either drug alone $(p<0.0001$, Figure 4B). The staining for the nuclear-specific antigen Ki67 revealed a significantly lower rate of proliferation in combination-treated H460 tumors $(p<0.0001$, Figure 4C). Pemetrexed-induced TS expression in tumors was suppressed by rapamycin $(p<0.05$, Figure 4D), and 4EBP1 phosphorylation was decreased by the combination. A further decrease in phosphorylation of S6 was not observed in tumor tissues, which is likely the result of effective inhibition of S6 phosphorylation by rapamycin alone. In $\mathrm{H} 157$ tumors, the combination of rapamycin and pemetrexed was also effective, decreasing growth by nearly $80 \%(p<0.0001$, Figure 4E) and inhibiting cellular proliferation $(p<0.0001$, Figure $4 \mathrm{~F})$. As observed in H460 tumors, rapamycin also suppressed pemetrexed-induced TS expression as assessed by immunohistochemistry $(p<0.05$, Figure 4G) (immunoblotting was not possible due to fixation of the tumors). These data suggest that rapamycin improves the inhibition of NSCLC tumor growth by preventing pemetrexed-induced expression of TS.

\section{DISCUSSION}

We investigated new approaches to enhance the efficacy of pemetrexed, and found that the combination of rapamycin and pemetrexed showed synergistic antiproliferative effects in NSCLC cells and enhanced mTOR inhibition, as demonstrated by decreases in the phosphorylation of the downstream components of this pathway such as 4E-BP1. Furthermore, rapamycin prevented pemetrexed-induced TS expression in vitro and in vivo. To our knowledge, this is the first study to describe the benefit of using an mTOR inhibitor to suppress the induction of TS that occurs as a compensatory response to pemetrexed in lung cancer model systems. Similar results have been reported in gastric cancer cell lines. Shigematsu, et al. reported that rapamycin decreased the endogenous expression levels of TS in gastric cancer cell lines, which enhanced chemotherapy-induced cytotoxicity [17]. Additionally, RAD001, a derivative of rapamycin, also decreased endogenous TS protein levels in gastric cancer cell lines. The combination of RAD001 with the anti-metabolite 5-fluorouracil significantly decreased TS protein levels to a greater extent than with RAD001 alone [18]. Collectively, these studies suggest that in general, mTOR inhibition is an effective way to decrease TS expression and increase the response to pemetrexed.

Despite the efficacy of the combination in the NSCLC cell lines we tested, our results are in conflict with those of Markova et al. [19], who showed that RAD001 protected NSCLC cells from pemetrexed-

A

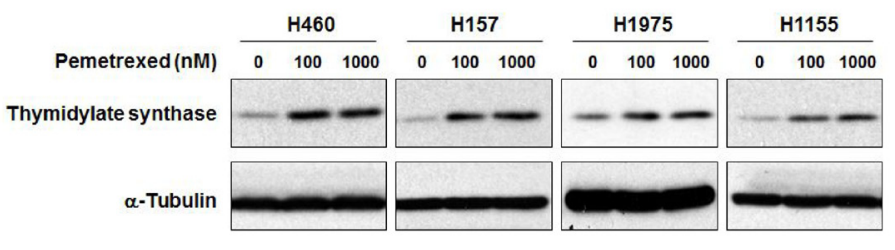

B

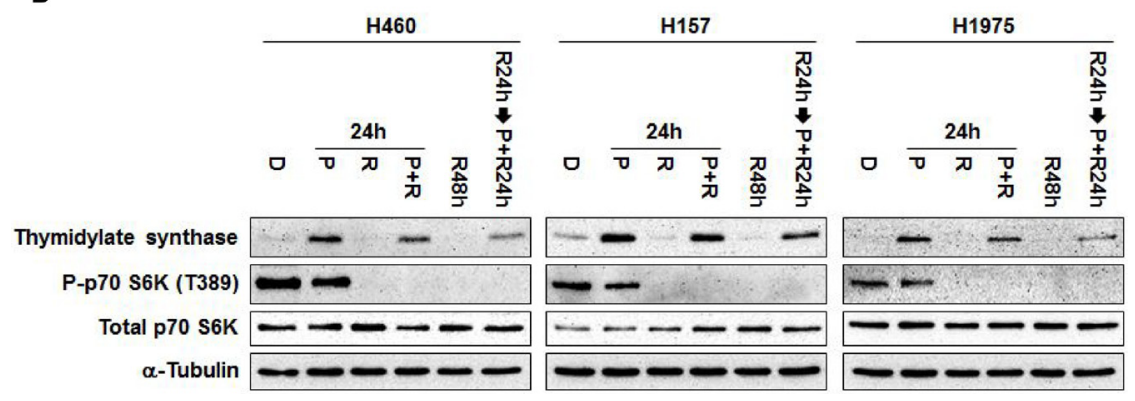

Figure 3: Rapamycin prevents pemetrexed-induced increases in TS expression. (A) Pemetrexed induces the expression of TS. NSCLC cells were treated with pemetrexed at the indicated concentrations for $24 \mathrm{hr}$. Levels of TS expression were assessed by immunoblotting analysis. (B) Pretreatment of cells with rapamycin improves its ability to suppress pemetrexed-induced increases in TS expression. D: $0.1 \%$ DMSO for $48 \mathrm{hr}$; P: $500 \mathrm{nM}$ pemetrexed for $24 \mathrm{hr}$; R: $100 \mathrm{nM}$ rapamycin for $24 \mathrm{hr}$; R48h: $100 \mathrm{nM}$ rapamycin for 48 $\mathrm{hr} ; \mathrm{R} 24 \mathrm{~h} \rightarrow \mathrm{P}+\mathrm{R} 24 \mathrm{~h}$ : pretreatment with $100 \mathrm{nM}$ rapamycin for $24 \mathrm{hr}$ followed by combining $500 \mathrm{nM}$ pemetrexed with $100 \mathrm{nM}$ rapamycin for $24 \mathrm{hr}$. Immunoblotting analysis was performed for the indicated biomarkers. 
A

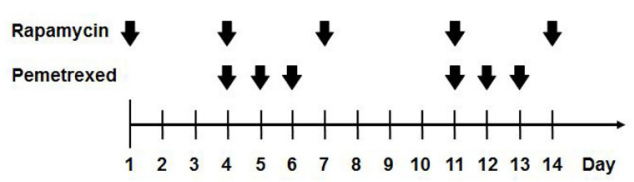

B

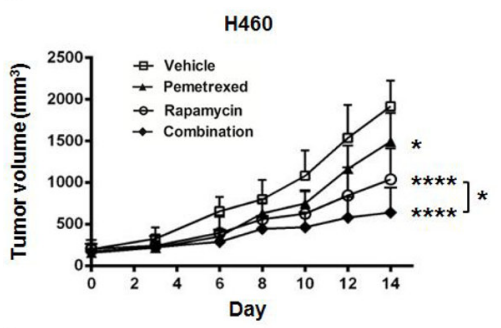

C

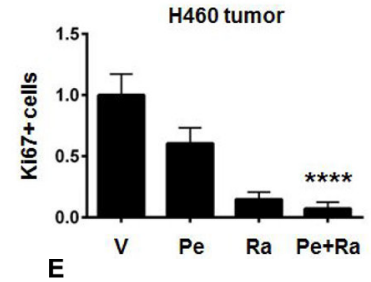

D

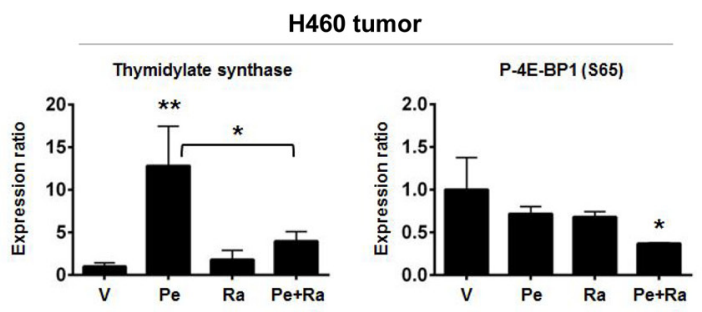

P-S6 (\$235/236)
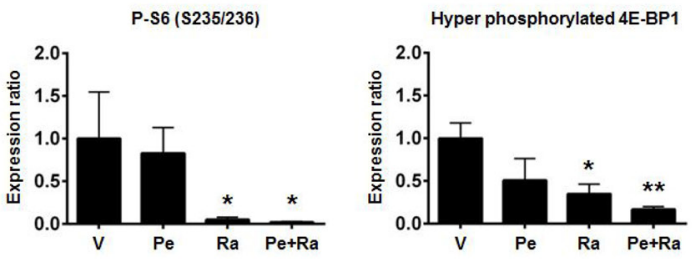

G

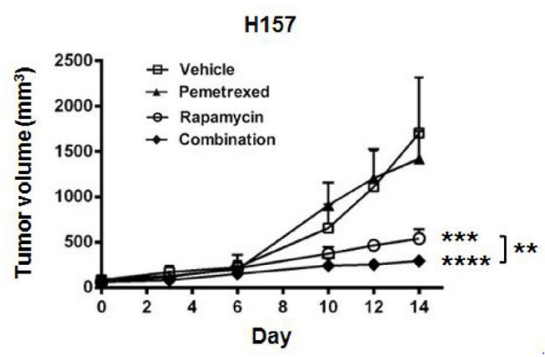

$\mathbf{F}$

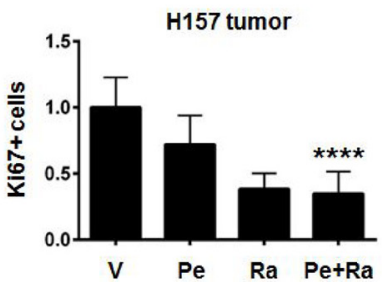

H157 tumor

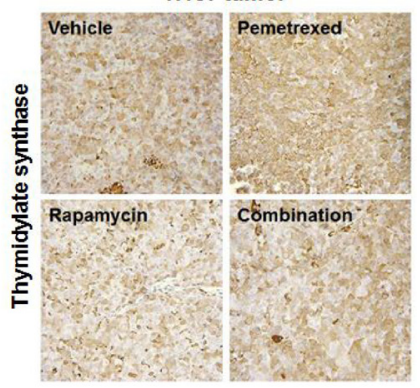

Thymidylate synthase

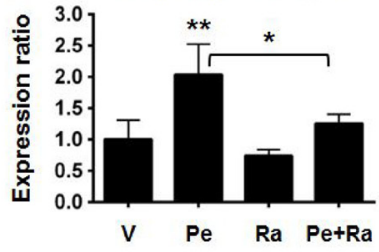

Figure 4: Combining rapamycin with pemetrexed inhibits the growth of NSCLC xenograft tumors to a greater extent than either drug alone. (A) The schema for NSCLC xenograft tumor treatments in vivo shows a lead in period of rapamycin alone followed by treatment with both rapamycin and pemetrexed. (B) H460 cells were grown as xenografts in athymic NCr-nu/nu. bars, SD. $*, p<0.05 ; * * *, p<0.0001$ for vehicle treatment. (C) Inhibition of cell proliferation in $\mathrm{H} 460$ tumors was assessed by immunohistochemistry for Ki67. The staining index was determined as described in Materials and Methods, and then normalized to vehicle for each treatment. Columns, mean from three of five mice examined in H460 xenografts. bars, SD. ****, p $<0.0001$ for vehicle treatment. (D) Biomarker analysis for the combination of rapamycin and pemetrexed in vivo. At the end of the study, H460 xenograft tumors were excised and processed for immunoblotting analysis of the indicated markers as described in Materials and Methods. Densitometry was performed using ImageJ version 1.43 software (http://rsbweb.nih.gov/ij/index.html). The levels of each marker were normalized to $\alpha$-Tubulin for each sample. Columns, show mean values from 3 mice. bars, SD. *, $\mathrm{p}<0.05 ;{ }^{* *}, \mathrm{p}<0.01$ for vehicle treatment. (E) H157 cells were grown as xenografts in athymic NCr-nu/nu. bars, SD. ${ }^{* *}, \mathrm{p}<0.01$ (unpaired $\mathrm{t}$ test); ${ }^{* * *}, \mathrm{p}<0.001$; ${ }^{* * *}, \mathrm{p}<0.0001$ for vehicle treatment. (F) Inhibition of cell proliferation in $\mathrm{H} 157$ tumors. Ki67+ cells were assessed by immunohistochemistry. The staining index was determined as described in Materials and Methods, and then normalized to vehicle for each treatment. Columns, mean from three of five mice examined in H157 xenografts. bars, SD. ****, p $<0.0001$ for vehicle treatment. (G) Rapamycin suppresses pemetrexed-induced increases in TS expression in vivo. Levels of TS were assessed by immunohistochemistry of H157 xenograft tumors excised at the end of the study. The TS staining index was determined as described in Materials and Methods, and then normalized to vehicle for each treatment. Columns, mean from all 5 mice examined in $\mathrm{H} 157$ xenografts. bars, SD. ${ }^{*}, \mathrm{p}<0.05 ;{ }^{* *}, \mathrm{p}<0.01$ for vehicle treatment. 
induced apoptosis. They concluded that mTOR inhibitors might suppress the antitumor activity of pemetrexed by slowing cell cycle progression. What might explain this discrepancy? In our study, the combination of rapamycin and pemetrexed showed synergistic effects in H460 and H1975 cells that have PIK3CA (phosphatidylinositol-4,5bisphosphate 3-kinase, catalytic subunit alpha) mutations, and in $\mathrm{H} 157$ and $\mathrm{H} 1155$ cells that have PTEN (phosphatase and tensin homolog) mutations. Each of these mutations leads to increased mTOR activity. In contrast, the NSCLC cell lines used by Markova et al. do not have PIK3CA or PTEN mutations. To further evaluate PIK3CA or PTEN mutations as predictive for response to the combination, we evaluated the effects of combining rapamycin with pemetrexed with other human NSCLC cell lines that do not have PIK3CA or PTEN mutations (H1703 and PC-9 (formerly known as PC-14) cells). Rapamycin did not enhance the efficacy of pemetrexed in these cell lines (data not shown). This is consistent with the results of MericBernstam et al., who reported that human cancer cells with PIK3CA and/or PTEN mutations were more likely to be sensitive to rapamycin [20]. Taken together, these findings suggest that PIK3CA or PTEN mutations contribute to the synergistic effects of combining rapamycin with pemetrexed.

Several reports show that pemetrexed-resistant lung cancer cell lines treated for 2 to 6 months have significantly increased levels of TS protein compared to the parental cell lines $[8,21]$. On the other hand, we found that short-term pemetrexed treatment induced TS expression, which was suppressed by rapamycin. Pretreatment of cells with rapamycin prior to administration of pemetrexed enhanced the suppression of pemetrexed-induced TS expression compared to the combination without rapamycin-pretreatment (Figure 3B). What mechanisms might underlie inhibition of TS by rapamycin? Because rapamycin inhibits protein synthesis, it is likely to suppress the translation of ribosomeassociated TS transcripts that are induced by pemetrexed. In addition, Lee et al. reported that the mechanism of TS protein down-regulation by RAD001 might be dependent on proteasomal degradation, because the treatment of a proteasome inhibitor blocked the down-regulation of TS protein by RAD001 [18]. Future studies might clarify the roles of the each of these mechanisms and/or identify additional mechanisms of TS suppression by mTOR inhibition.

The results of these studies have clinical implications. TS may be a valuable target in NSCLC. For example, Kaira et al. reported that positive TS expression in tumor tissues from 160 patients with completely resected NSCLC was significantly associated with advanced stage and lymph node metastases, and was an independent prognostic factor for predicting a poor outcome in patients with lung adenocarcinoma [22]. A recent meta-analysis that evaluated the predictive value of
TS in pemetrexed-containing chemotherapy regimens for NSCLC patients suggests that increased levels of TS are an independent risk factor for potential resistance against pemetrexed [10]. Early clinical trials combining mTOR inhibitors with pemetrexed have been completed. A phase I study of RAD001 given simultaneously with pemetrexed in previously treated NSCLC patients has been reported, and the regimen was well tolerated with a $42 \%$ disease control rate (partial response (PR) and stable disease) [23]. These results are similar to what might be expected for pemetrexed alone, but biomarkers and TS levels were not analyzed in this study. A hypothetical advantage of using rapamycin includes the fact that rapamycin can protect normal cells from the cytotoxicity of chemotherapeutic agents and radiation-induced damage, but sensitize cancer cells to these therapeutic modalities $[24,25]$. We recently completed a Phase I/II clinical trial combining rapamycin and pemetrexed in patients with relapsed NSCLC who had any number of prior treatments, and found that the regimen was well tolerated with a $22 \%$ PR rate (manuscript in preparation). We incorporated a one-week lead in period with rapamycin alone for pharmacokinetics and biomarker analysis. Immunoblotting analysis of peripheral blood mononuclear cells collected from patients showed that rapamycin alone decreased the level of endogenous expression of TS, which correlated with progression free survival and was consistent with our preclinical results [26]. These results suggest that the maximum clinical benefit of combining mTOR inhibition with pemetrexed might be achieved through a lead in period with mTOR inhibition alone. In addition, future studies should assess basal levels of TS expression in tumor tissue and stratify patients based on status of PIK3CA and PTEN to validate potential predictive markers.

\section{MATERIALS AND METHODS}

\section{Cell culture.}

NSCLC (H460, H157, and H1155) cell lines with Kras mutations were established at the National Cancer Institute (NCI, Bethesda, MD, USA), and the NSCLC H1975 cell line with EGFR mutations was a kind gift from Dr. William Pao (Vanderbilt-Ingram Cancer Center, Nashville, TN, USA). All cell lines were maintained in $75 \mathrm{~cm} 2$ flask in RPMI 1640 (Life Technologies, Grand Island, NY, USA) with $5 \%$ fetal bovine serum (FBS, Life Technologies) at $37 \circ \mathrm{C}$ in a $5.0 \% \mathrm{CO} 2$ atmosphere incubator. LKB1 mutations are detected in $\mathrm{H} 460$ and H157 cell lines. The mutation status of NSCLC cells was obtained from Cell Line Project, Catalog of Somatic Mutations in Cancer (COSMIC), the Wellcome Trust Sanger Institute Cancer Genome Project website (http:// cancer.sanger.ac.uk/cancergenome/projects/cosmic/). 


\section{Reagents.}

Rapamycin and pemetrexed were obtained from LC Laboratories (Woburn, MA, USA). Primary antibodies for P-4E-BP1 (Ser65), 4E-BP1, P-S6 Ribosomal Protein (Ser235/236), S6 Ribosomal Protein, thymidylate synthase (TS), P-p70 S6 Kinase (Thr389), and p70 S6 Kinase were from Cell Signaling Technology (Beverly, MA, USA). Anti- $\alpha$-Tubulin and Ki67 antibodies were from Sigma (St. Louis, MO, USA) and Abcam (Cambridge, MA, USA), respectively.

\section{Cell proliferation assay and Combination Index (CI).}

NSCLC cells $(2,500$ cells per well) were plated in 96-well plates and allowed to grow overnight. Cells were treated with rapamycin dissolved in DMSO, pemetrexed dissolved in PBS, or the combination in a wide concentration range between $0.01 \mathrm{nM}$ and 10000 $\mathrm{nM}$ for $72 \mathrm{hr}$ (H460 and H1155 cells) or $96 \mathrm{hr}$ (H157 and H1975 cells). Growth inhibition was determined by the sulforhodamine B assay [27]. Percent growth value was calculated by using the absorbance values of untreated cells on day 0 (D0), DMSO-treated control cells $(\mathrm{C})$, and drug-treated cells $(\mathrm{T})$ as follows: [(T - D0)/ (C - D0)] x 100 for concentrations for which $\mathrm{T}>/=\mathrm{D} 0$, or $[(\mathrm{T}-\mathrm{D} 0) / \mathrm{C}] \times 100$ for concentrations for which $\mathrm{T}<\mathrm{D} 0$. Experiments were performed three times, and each drug concentration was evaluated in sextuplet wells for a given experiment. CI is a quantitative measure of the degree of drug interaction in terms of synergism $(\mathrm{CI}<1)$, additive effect $(\mathrm{CI}=1)$, or antagonism $(\mathrm{CI}>1)$ for a given endpoint of the effect measurement [28]. Additive effect is defined as the combined effect predicted by the massaction law principle, synergism is as the production of a greater than expected additive effect, and antagonism is as the production of smaller than expected additive effect. Inhibitory concentration 50 (IC50) and Combination Indices (CIs) were calculated using CompuSyn software program (ComboSyn, Inc., Paramus, NJ, USA). Affected fraction $(\mathrm{Fa})$ is defined as a function of effect level (e.g., degree of inhibition) by a dose of drug. Fa values were calculated according to the program's instruction as follows: [(100 - \% growth value $) / 100]$, which indicated a growth inhibition value.

\section{Immunoblotting.}

Cells $\left(5 \times 10^{5}\right.$ cells per well) were plated in sixwell plates. The following day, cells were treated with drug or equal volume of DMSO for the indicated times and lysed in 2 x lysis buffer as described previously [29]. For tumor-tissue homogenates in vivo, frozen tumors were allowed to thaw on ice, then homogenized in radioimmunoprecipitation assay buffer $[150 \mathrm{mmol} / \mathrm{L}$ $\mathrm{NaCl}, 1 \%$ Igepal CA-630, $0.5 \%$ sodium deoxycholate, $0.1 \% \mathrm{SDS}, 50 \mathrm{mmol} / \mathrm{L}$ Tris $(\mathrm{pH} 8.0)]$ containing $2.5 \mathrm{~mol} / \mathrm{L}$ h-glycerol phosphate, $0.2 \mathrm{~mol} / \mathrm{L}$ sodium orthovanadate, $1.25 \mathrm{~mol} / \mathrm{L}$ sodium fluoride, and $1 \mathrm{x}$ protease inhibitor cocktail (Roche Diagnostics, Indianapolis, IN, USA) using a hand-held Tissue-Tearor homogenizer (Biospec Products, Bartlesville, OK, USA). Cell lysates or tumortissue homogenates with equal amounts of protein were separated by SDS-PAGE then transferred to nitrocellulose membranes. The membranes were blocked for $1 \mathrm{hr}$ in blocking buffer ( 1 x TBS, $5 \%$ milk, $0.1 \%$ Tween 20 ) and placed in primary antibody diluted in $1 \times \mathrm{TBS}, 5 \%$ bovine serum albumin, $0.1 \%$ Tween 20 , overnight at $4^{\circ} \mathrm{C}$. The following day, membranes were washed thrice in wash buffer $(0.1 \%$ Tween $20,1 \times$ TBS $)$. Primary antibody was detected using horseradish peroxidaselinked secondary antibodies and visualized with the enhanced chemiluminescent detection system (Amersham Biosciences, Pittsburgh, PA, USA). Immunoblot experiments were performed at least 3 times.

\section{Drug treatment in vivo.}

Six-week-old female athymic NCr-nu/nu mice (Charles River Labs, Frederick, MD, USA) were injected subcutaneously (s.c.) in both rear flanks with $5 \times 10^{6} \mathrm{H} 460$ or H157 cells in $100 \mu \mathrm{l}$ PBS. When the transplanted tumors reached a volume of $50 \mathrm{~mm}^{3}$, mice were divided into the following four groups ( 5 mice per group): intraperitoneal (i.p.) injection of either (1) vehicle (4\% DMSO, 5\% PEG, $5 \%$ Tween 80 in saline; once daily on days 1,4 to 7 , and 11 to 14 ), (2) $2.5 \mathrm{mg} / \mathrm{kg}$ rapamycin (once daily on days 1 , $4,7,11$, and 14) as described previously [14], (3) $100 \mathrm{mg} /$ $\mathrm{kg}$ pemetrexed (once daily on days 4 to 6 and 11 to 13) based on tolerated dosage as described previously [30], or (4) the combination of rapamycin and pemetrexed (once daily on each drug's schedule). Animal weights and tumor measurements were made every other day. In all studies, tumor volume was calculated from the formula $\mathrm{v}=\left(a b^{2}\right) /$ 2 , where $a$ is the long axis and $b$ is the short axis. In vivo experiments were conducted under a protocol approved by the NCI Animal Care and Use Committee.

\section{Immunohistochemistry.}

Formalin-fixed, paraffin-embedded xenograft tumor tissues were sectioned, placed on poly-Llysine-coated slides (Histoserv Inc., Germantown, MD, USA), and analyzed for protein expression for five mice per group. Antigen retrieval was carried out using preheated target retrieval solution $(\mathrm{pH}$ 6.0) from DakoCytomation (Carpinteria, CA, USA) for $30 \mathrm{~min}$ in a boiling rice cooker. Vectastain Elite $\mathrm{ABC}$ kits from 
Vector Laboratories (Burlingame, CA, USA) were used according to manufacturer's instructions for blocking, dilution of primary antibody, and labeling. Primary antibody was incubated with sections for $16 \mathrm{hr}$ at $4^{\circ} \mathrm{C}$. 3,3- Diaminobenzidine was prepared fresh from tablets (Sigma). Specificity of staining was assessed by comparison with samples stained in the absence of primary antibody. All slides were blinded to the investigators before scoring, and in all cases, xenograft tumors were assessed for three to five mice per group. The staining index of TS was determined by assigning a score of absent (0), minimal (1), moderate (2), or high (3) staining to each cell in five 400-highpower fields (HPF). The staining index was then calculated by multiplying the staining intensity by its distribution scored as $0(0 \%), 1$ ( $1 \%$ to $20 \%), 2(21 \%$ to $40 \%), 3$ (41\% to $60 \%), 4(61 \%$ to $80 \%), 5(81 \%$ to $100 \%)$ to indicate the percentage of positive cells of interest in a single core. Ki67 staining was quantified by counting the number of the positive cells in five 400-HPF per tumor. Numbers were averaged for three to five mice per group. An overall score was assigned to each slide, and the scores were averaged for vehicle versus drug-treated groups.

\section{Statistics analysis.}

Statistical significance of differences observed in drug-treated and untreated cells was analyzed using oneway/two-way ANOVA, and then multiple comparisons were performed by Bonferroni test. All analyses were performed using the GraphPad Prism software version $5.0 \mathrm{c}$. The threshold value was set to 0.05 .

\section{ACKNOWLEDGEMENTS}

This research was supported by the Intramural Research Program of the NIH, Center for Cancer Research, National Cancer Institute. The content of this publication does not necessarily reflect the views or policies of the Department of Health and Human Services, nor does mention of trade names, commercial products, or organization imply endorsement by the United States Government. Publication of this article was funded in part by the Open Access Promotion Fund of the Johns Hopkins University Libraries.

\section{REFERENCES}

1. Siegel R, Naishadham D and Jemal A. Cancer statistics, 2013. CA Cancer J Clin. 2013; 63(1):11-30.

2. Howlader N, Noone AM, Krapcho M, Garshell J, Neyman N, Altekruse SF, Kosary CL, Yu M, Ruhl J, Tatalovich Z, Cho H, Mariotto A, Lewis DR, Chen HS, Feuer EJ and Cronin KA. SEER Cancer Statistics Review, 1975-2010, National Cancer Institute. Bethesda, MD. 2013.
3. Ettinger DS, Akerley W, Borghaei H, Chang AC, Cheney RT, Chirieac LR, D’Amico TA, Demmy TL, Ganti AK, Govindan R, Grannis FW, Jr., Horn L, Jahan TM, Jahanzeb $\mathrm{M}$, Kessinger A, Komaki R, et al. Non-small cell lung cancer. J Natl Compr Canc Netw. 2012; 10(10):1236-1271.

4. Joerger M, Omlin A, Cerny T and Fruh M. The role of pemetrexed in advanced non small-cell lung cancer: special focus on pharmacology and mechanism of action. Curr Drug Targets. 2010; 11(1):37-47.

5. Peters S, Adjei AA, Gridelli C, Reck M, Kerr K, Felip $\mathrm{E}$ and Group EGW. Metastatic non-small-cell lung cancer (NSCLC): ESMO Clinical Practice Guidelines for diagnosis, treatment and follow-up. Ann Oncol. 2012; 23 Suppl 7:vii56-64.

6. Socinski MA, Evans T, Gettinger S, Hensing TA, Sequist LV, Ireland B and Stinchcombe TE. Treatment of stage IV non-small cell lung cancer: Diagnosis and management of lung cancer, 3rd ed: American College of Chest Physicians evidence-based clinical practice guidelines. Chest. 2013; 143(5 Suppl):e341S-368S.

7. Gonen N and Assaraf YG. Antifolates in cancer therapy: structure, activity and mechanisms of drug resistance. Drug Resist Updat. 2012; 15(4):183-210.

8. Ozasa H, Oguri T, Uemura T, Miyazaki M, Maeno K, Sato $\mathrm{S}$ and Ueda R. Significance of thymidylate synthase for resistance to pemetrexed in lung cancer. Cancer Sci. 2010; 101(1):161-166.

9. Takezawa K, Okamoto I, Okamoto W, Takeda M, Sakai K, Tsukioka S, Kuwata K, Yamaguchi H, Nishio K and Nakagawa K. Thymidylate synthase as a determinant of pemetrexed sensitivity in non-small cell lung cancer. Br J Cancer. 2011; 104(10):1594-1601.

10. Liu Y, Yin TJ, Zhou R, Zhou S, Fan L and Zhang RG. Expression of thymidylate synthase predicts clinical outcomes of pemetrexed-containing chemotherapy for non-small-cell lung cancer: a systemic review and metaanalysis. Cancer Chemother Pharmacol. 2013; 72(5):11251132.

11. Laplante $M$ and Sabatini DM. mTOR signaling in growth control and disease. Cell. 2012; 149(2):274-293.

12. Racanelli AC, Rothbart SB, Heyer CL and Moran RG. Therapeutics by cytotoxic metabolite accumulation: pemetrexed causes ZMP accumulation, AMPK activation, and mammalian target of rapamycin inhibition. Cancer Res. 2009; 69(13):5467-5474.

13. Rothbart SB, Racanelli AC and Moran RG. Pemetrexed indirectly activates the metabolic kinase AMPK in human carcinomas. Cancer Res. 2010; 70(24):10299-10309.

14. Granville CA, Warfel N, Tsurutani J, Hollander MC, Robertson M, Fox SD, Veenstra TD, Issaq HJ, Linnoila RI and Dennis PA. Identification of a highly effective rapamycin schedule that markedly reduces the size, multiplicity, and phenotypic progression of tobacco carcinogen-induced murine lung tumors. Clin Cancer Res. 
2007; 13(7):2281-2289.

15. Frings V, van der Veldt AA, Boellaard R, Herder GJ, Giovannetti E, Honeywell R, Peters GJ, Thunnissen E, Hoekstra OS and Smit EF. Pemetrexed induced thymidylate synthase inhibition in non-small cell lung cancer patients: a pilot study with 3'-deoxy-3'-[(1)(8)F]fluorothymidine positron emission tomography. PLoS One. 2013; 8(5):e63705.

16. Ceppi P, Rapa I, Lo Iacono M, Righi L, Giorcelli J, Pautasso M, Bille A, Ardissone F, Papotti M and Scagliotti GV. Expression and pharmacological inhibition of thymidylate synthase and Src kinase in nonsmall cell lung cancer. Int J Cancer. 2012; 130(8):1777-1786.

17. Shigematsu H, Yoshida K, Sanada Y, Osada S, Takahashi T, Wada Y, Konishi K, Okada M and Fukushima M. Rapamycin enhances chemotherapy-induced cytotoxicity by inhibiting the expressions of TS and ERK in gastric cancer cells. Int J Cancer. 2010; 126(11):2716-2725.

18. Lee KH, Hur HS, Im SA, Lee J, Kim HP, Yoon YK, Han SW, Song SH, Oh DY, Kim TY and Bang YJ. RAD001 shows activity against gastric cancer cells and overcomes 5-FU resistance by downregulating thymidylate synthase. Cancer Lett. 2010; 299(1):22-28.

19. Markova B, Hahnel PS, Kasper S, Herbertz S, Schuler M and Breitenbuecher F. Pharmacologic inhibition of mTOR antagonizes the cytotoxic activity of pemetrexed in nonsmall cell lung cancer. J Cancer Res Clin Oncol. 2012; 138(4):545-554.

20. Meric-Bernstam F, Akcakanat A, Chen H, Do KA, Sangai T, Adkins F, Gonzalez-Angulo AM, Rashid A, Crosby K, Dong M, Phan AT, Wolff RA, Gupta S, Mills GB and Yao J. PIK3CA/PTEN mutations and Akt activation as markers of sensitivity to allosteric mTOR inhibitors. Clin Cancer Res. 2012; 18(6):1777-1789.

21. Zhang D, Ochi N, Takigawa N, Tanimoto Y, Chen Y, Ichihara E, Hotta K, Tabata M, Tanimoto M and Kiura K. Establishment of pemetrexed-resistant non-small cell lung cancer cell lines. Cancer Lett. 2011; 309(2):228-235.

22. Kaira K, Ohde Y, Nakagawa K, Okumura T, Murakami $\mathrm{H}$, Takahashi $\mathrm{T}$, Kondo H, Nakajima T, Endo $\mathrm{M}$ and Yamamoto N. Thymidylate synthase expression is closely associated with outcome in patients with pulmonary adenocarcinoma. Med Oncol. 2012; 29(3):1663-1672.

23. Vansteenkiste J, Solomon B, Boyer M, Wolf J, Miller N, Di Scala L, Pylvaenaeinen I, Petrovic K, Dimitrijevic S, Anrys B and Laack E. Everolimus in combination with pemetrexed in patients with advanced non-small cell lung cancer previously treated with chemotherapy: a phase I study using a novel, adaptive Bayesian dose-escalation model. J Thorac Oncol. 2011; 6(12):2120-2129.

24. Apontes P, Leontieva OV, Demidenko ZN, Li F and Blagosklonny MV. Exploring long-term protection of normal human fibroblasts and epithelial cells from chemotherapy in cell culture. Oncotarget. 2011; 2(3):222233.
25. Iglesias-Bartolome R, Patel V, Cotrim A, Leelahavanichkul K, Molinolo AA, Mitchell JB and Gutkind JS. mTOR inhibition prevents epithelial stem cell senescence and protects from radiation-induced mucositis. Cell Stem Cell. 2012; 11(3):401-414.

26. Blumenthal GM, Ballas MS, Bernstein W, Shamloo BK, Root H, Helsabeck C, Chun G, Figg WD, Giaccone G and Dennis PA. A phase I/II trial of pemetrexed and sirolimus in advanced NSCLC. J Clin Oncol 2010; 28:15s (suppl; abstr 7600)

27. Skehan P, Storeng R, Scudiero D, Monks A, McMahon J, Vistica D, Warren JT, Bokesch H, Kenney S and Boyd MR. New colorimetric cytotoxicity assay for anticancer-drug screening. J Natl Cancer Inst. 1990; 82(13):1107-1112.

28. Chou TC. Theoretical basis, experimental design, and computerized simulation of synergism and antagonism in drug combination studies. Pharmacol Rev. 2006; 58(3):621681.

29. Kawabata S, Gills JJ, Mercado-Matos JR, Lopiccolo J, Wilson W, 3rd, Hollander MC and Dennis PA. Synergistic effects of nelfinavir and bortezomib on proteotoxic death of NSCLC and multiple myeloma cells. Cell Death Dis. 2012; 3:e353.

30. Tonkinson JL, Worzalla JF, Teng CH and Mendelsohn LG. Cell cycle modulation by a multitargeted antifolate, LY231514, increases the cytotoxicity and antitumor activity of gemcitabine in HT29 colon carcinoma. Cancer Res. 1999; 59(15):3671-3676. 